\title{
$\delta$ Scuti and $\gamma$ Doradus stars
}

\author{
Michel Breger \\ Institute of Astronomy, University of Vienna, Türkenschanzstr. 17, A-1180 Wien, Austria \\ email: michel.breger@univie.ac.at
}

\begin{abstract}
The paper emphasizes the connection between observations and theory in the Lower Instability Strip. It is argued that the $\delta$ Scuti and $\gamma$ Doradus pulsators are very close relatives in the sense that the $\gamma$ Doradus phenomenon can also be seen in the slightly hotter $\delta$ Scuti variables.

New developments and recent progress are reviewed. Arguments are given why all (or almost all) stars in the Lower Instability Strip are pulsators. Furthermore, the question of the so-called missing modes is answered as very extensive photometry campaigns have revealed a large number of pulsation modes with amplitudes less than one millimag. Arguments are given that the $500+$ $\ell=0$ to 2 modes predicted for $\delta$ Scuti stars are actually present.

The progress made in developing reliable methods of pulsation mode identification is briefly discussed. The discovery of many close modes with small amplitudes sets severe requirements concerning the length of future studies.
\end{abstract}

Keywords. Stars: variables: $\delta$ Scuti, stars: oscillations (including pulsations)

\section{Introduction}

$\delta$ Scuti stars are pulsators situated in the Classical Instability Strip on and above the Main Sequence. They pulsate with radial and nonradial pressure (p) as well as mixed pressure/gravity $(\mathrm{p} / \mathrm{g})$ modes. The excitation is due to the $\kappa$ mechanism in the He II ionization zone.

$\gamma$ Doradus stars are gravity (g) mode pulsators. In the H-R Diagram they overlap the $\operatorname{cool} \delta$ Scuti stars and extend into the F stars. The details of the excitation are not entirely clear at this stage. Convective blocking has been proposed as a mechanism (Guzik et al. 2000, Dupret et al. 2004).

Despite their difference in the types of excited pulsation modes and the length of the pulsation periods, the two types of stars may be related. No known $\gamma$ Doradus star has so far been found to show short-period $\delta$ Scuti pulsation. The converse may not be true: many cool $\delta$ Scuti stars may show the $\gamma$ Doradus phenomenon, both from observations and theoretical models. This is illustrated in Fig. 1, where excitation as a function of frequency is shown for the $\ell=2$ modes of the $\delta$ Scuti star FG Vir. The instability parameter, $\eta$, shows a second peak in the low-frequency region, viz., near $1.7 \mathrm{~cd}^{-1}$. The peak remains negative, but varies with the convection treatment adopted. It is attractive to speculate that the peak can actually become positive so that gravity modes can be excited in a limited part of the low-frequency region.

These low-frequency peaks have been observed in some $\delta$ Scuti stars. Attempts to explain them in terms of other hypotheses such as combination modes have failed. An example is the star BI CMi (Breger et al. 2002). This star pulsates in the 4.8 to 13.0 $\mathrm{c} \mathrm{d}^{-1}$ region, but also shows a clear peak at $1.662 \mathrm{c} \mathrm{d}^{-1}$. Fig. 2 demonstrates this relative to the main comparison star, HD 66925. We note here that the peak is also seen relative to a second comparison star, HD 66829. 

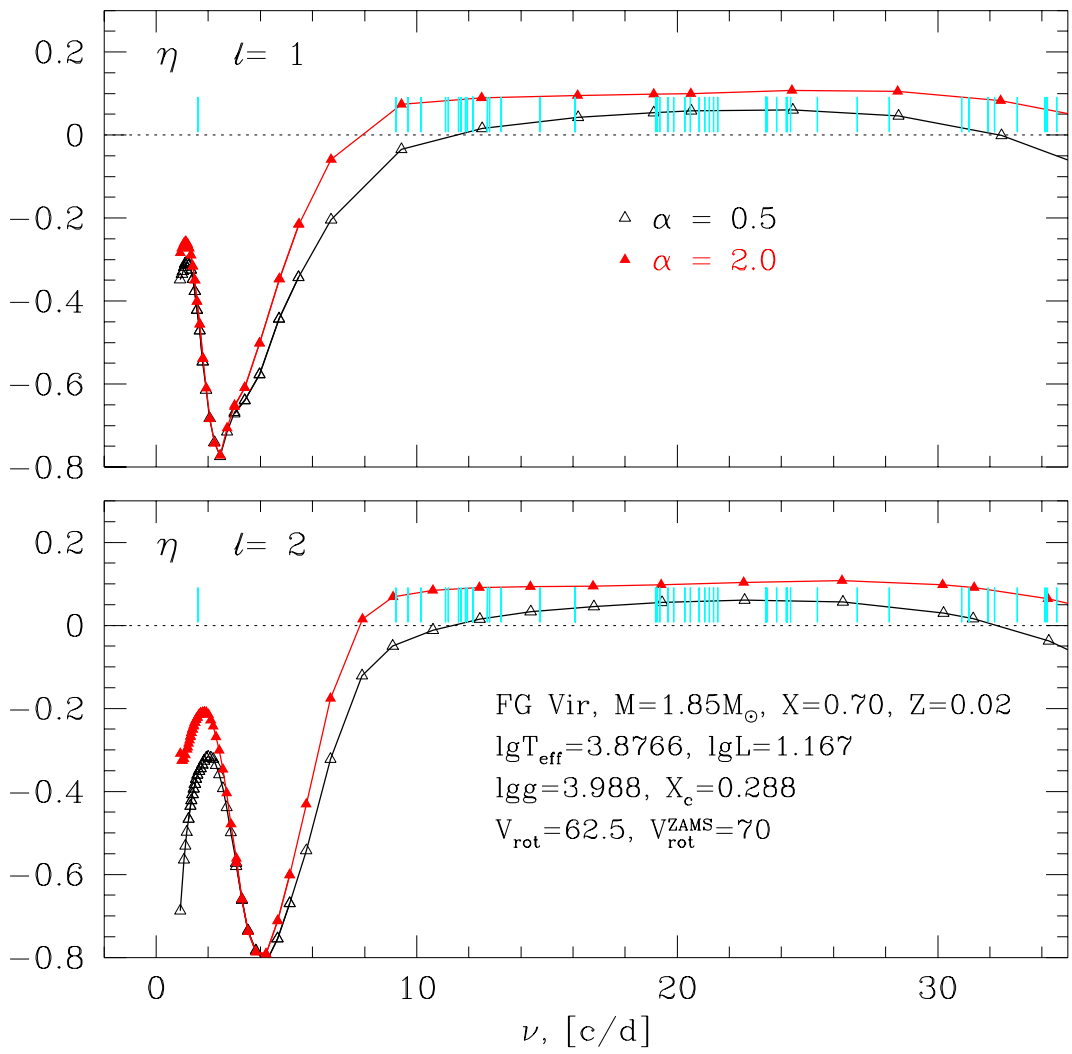

Figure 1. Pulsation excitation models calculated by A. A. Pamyatnykh for a typical evolved $\delta$ Scuti star. The parameter $\eta$ denotes the amount of instability. The two curves were calculated with $\alpha$ values of 0.5 (bottom) and 2.0 (top). Instability occurs above the horizontal line and is in agreement with the observed frequencies of FG Vir. The local maximum at low frequencies may point towards an explanation of the observed low-frequency peaks in FG Vir and other $\delta$ Scuti variables.

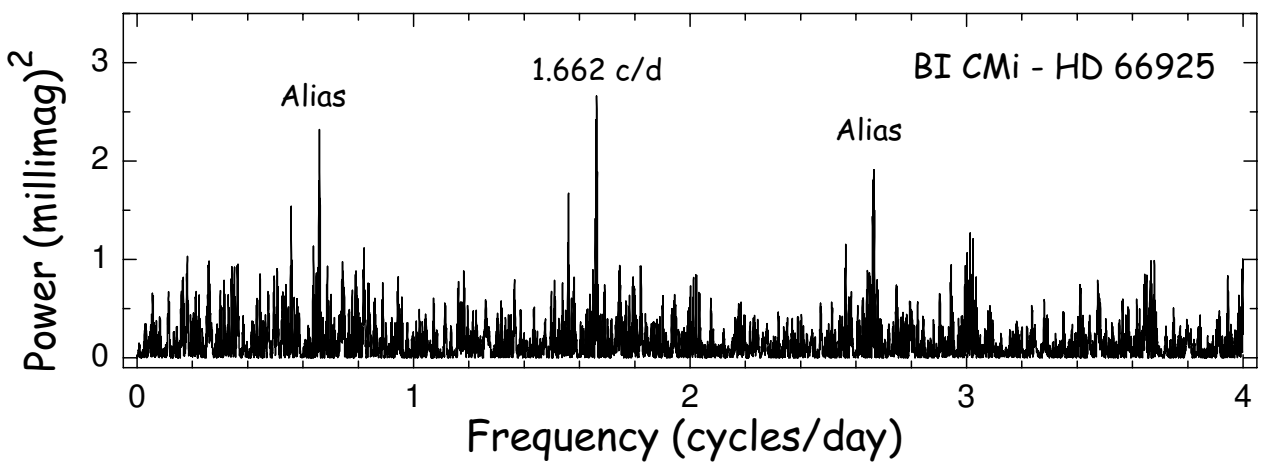

Figure 2. Power spectrum of the cool $\delta$ Scuti star BI CMi in the low-frequency region. The peak at $1.662 \mathrm{c} \mathrm{d}^{-1}$ could be interpreted as a gravity mode, if the local excitation maximum near $1.7 \mathrm{~cd}^{-1}$ shown by some models is strong enough.

\section{What fraction of stars in the Lower Instability Strip are variable?}

A number of photometric variability surveys of stars in and near the Lower Instability Strip have detected variability in about $25 \%-33 \%$ of the stars. The results depend on 
the photometric precision (i.e., the amplitude threshold) and the width of the instability strip. A excellent recent discussion can be found in Poretti et al. (2003).

A much higher incidence of pulsation is obtained if one corrects for the observational limits of the surveys. If we examine the distribution of amplitudes of the $\delta$ Scuti variables found in surveys and extrapolate this to near-zero amplitude, all or maybe all the stars in the Lower Instability Strip should be pulsating.

This expectation is dramatically confirmed by new WIRE satellite measurements of the bright A star, Altair. These measurements are more accurate than ground-based photometry of equal observing time. (Later we will show that ground-based photometry can, and does detect, amplitudes of $0.2 \mathrm{mmag}$ ). Buzasi et al. (2004) show that Altair is variable with an amplitude of about 1 mmag. This confirms the expectation that most (or all) of the 'constant' stars in the Instability Strip are variable at small amplitudes.

What determines the amplitude of a $\delta$ Scuti or a $\gamma$ Doradus star? The largest effect is due to stellar rotation (see Fig. 3): high amplitude requires low rotation. Often, but not always, these high-amplitude modes are radial. Stars rotating faster than $\sim 30 \mathrm{~km} \mathrm{~s}^{-1}$ pulsate with a mixture of low-amplitude radial and nonradial modes. Why some of these amplitudes are as large as $0.02 \mathrm{mag}$. and some are as small $0.0002 \mathrm{mag}$. is not known at this time.

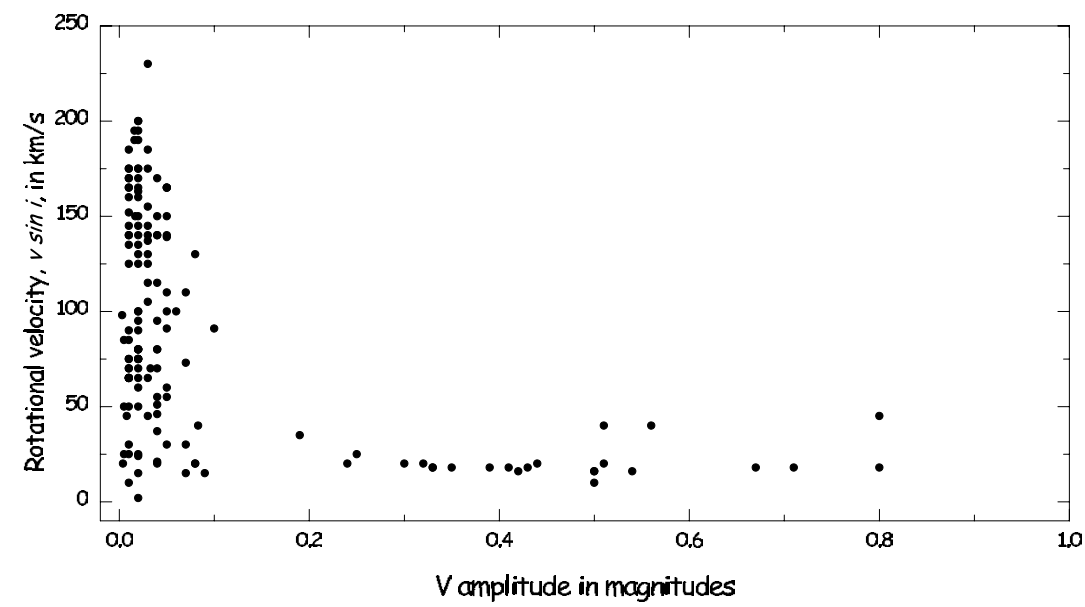

Figure 3. Relationship between observed photometric amplitude and measured rotational velocity, $v \sin i$. This diagram shows that high-amplitude pulsation is restricted to slow rotation.

\section{Pulsation mode identifications}

One of the most important prerequisites for the application of asteroseismology is the identification of the discovered frequencies with the stellar pulsation modes, i.e., the determination of pulsation quantum numbers $n, \ell, m$. A variety of photometric and spectroscopic techniques are applied. In the last few years, there has been enormous progress in the development and checking of these techniques. Space limitations do not permit more details here, but examples can be found in Aerts et al. (2004) and Zima et al. (2004). Apart from mode identifications, spectroscopic line-profile analyses can also be used to determine the inclination of the rotation axis to the line of sight. Fig. 4, kindly supplied by W. Zima, demonstrates this for the star FG Vir. 


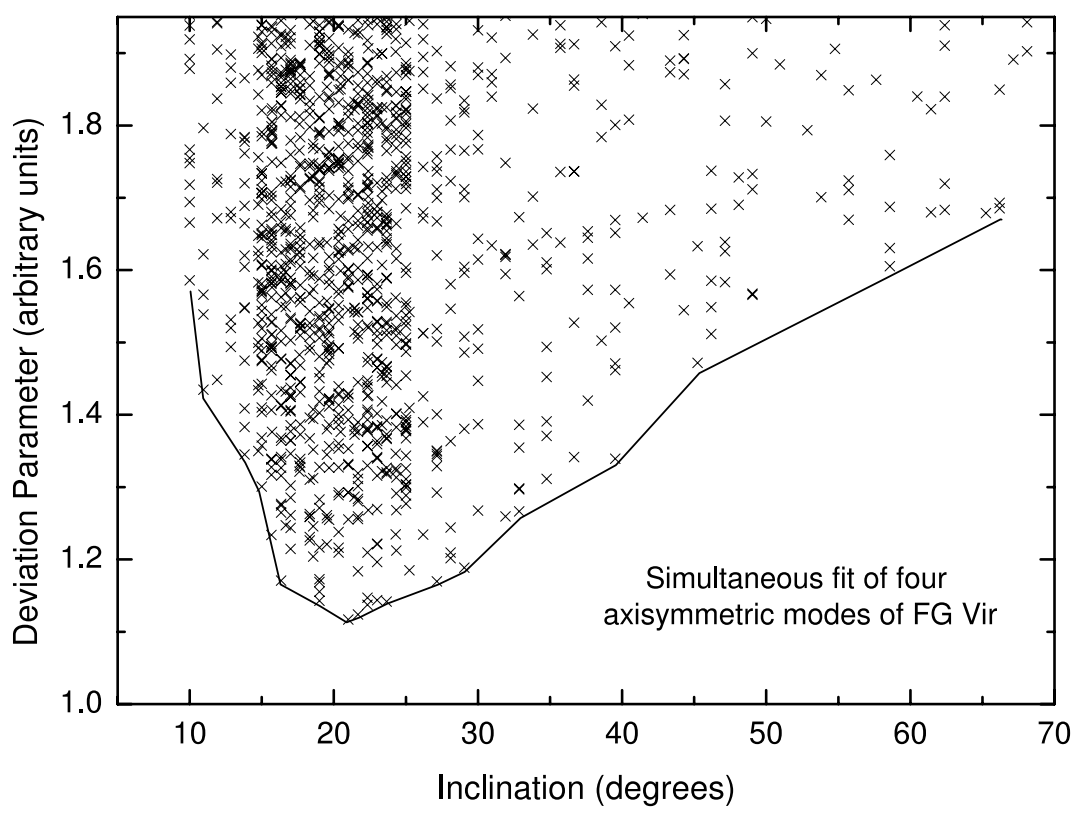

Figure 4. Line-profile variations can be used to also determine the inclination of the rotation axis to the line of sight. For the star FG Vir, the observed profile variations of four non-axisymmetric modes were fitted simultaneously with a common inclination. The diagram shows the quality of fits of more than 15000 simulations (some shown by crosses) to the observed line profiles of FG Vir. The best agreement is found for an angle of $20^{\circ}$.

\section{Where are the missing modes?}

$\delta$ Scuti star models predict pulsational instability in many radial and nonradial modes. The observed number of low-degree modes is much smaller than the number predicted by pulsation models. The problem of mode selection is most severe for post-Main-Sequence $\delta$ Scuti stars, which comprise about $40 \%$ of the observed $\delta$ Scuti stars. The theoretical frequency spectrum of unstable modes is very dense. Most modes are of mixed character as they behave like p-modes in the envelope and like g-modes in the interior. For example, a model of $4 \mathrm{CVn}$ predicts 554 unstable modes of $\ell=0$ to 2 . Of these 6 are $\ell=0,168$ are $\ell$ $=1$, and 380 are $\ell=2$. However, only 18 (and an additional 16 combination frequencies) were observed (Breger et al. 1999). The problem for other $\delta$ Scuti stars is similar.

Consequently, either the theoretical predictions or the observational techniques are imperfect (for a discussion, see Breger \& Pamyathykh 2002). On the observational side it is, therefore, necessary to lower the observational threshold to search for the missing modes. The answer may be provided by the $1000+$ hours of photometry obtained by the $\delta$ Scuti Network for FG Vir. The preliminary multifrequency solution already has identified 60 frequencies. The separate solutions for 2002 and 2003 are similar, so that we regard the results to be reliable.

The amplitude distribution is shown in Fig. 5. The diagram shows an almost exponential curve with decreasing amplitude. Since it is improbable that the star stops at exactly the observational threshold, considerably more modes should be present near 0.2 mmag.

This is supported by the power spectrum of the residuals after the 60-frequency solution is subtracted (Fig. 6). Excess power in the frequency regions, in which FG Vir is known to pulsate, indicates the existence of many additional modes. 


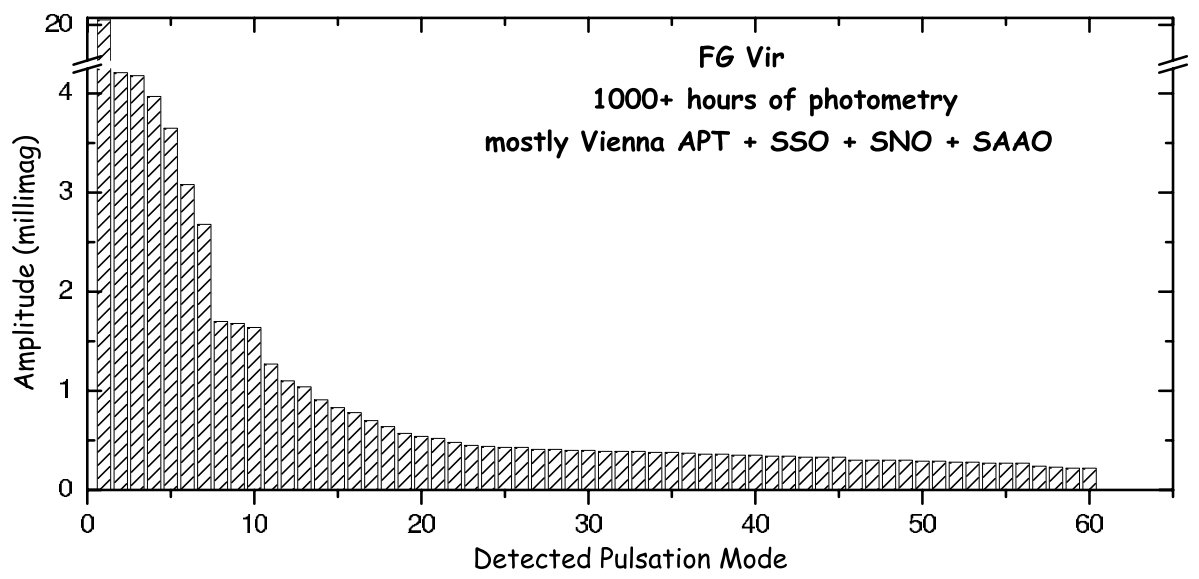

Figure 5. Photometric amplitudes of the pulsation modes of FG Vir. The detection of 60 modes is preliminary and based on 2002 and 2003 data of the $\delta$ Scuti Network. The exponential shape of the distribution suggests that below the observational limit $0.20 \mathrm{mmag}$ considerably more modes are present.

While we have not detected all of the $\sim 500$ predicted pulsation modes, the huge increase in detected modes with the low 0.20 mmag threshold strongly suggests that the predicted modes are not missing. While the question of mode selection may now be answered, the question of what determines the sizes of the amplitudes remains.

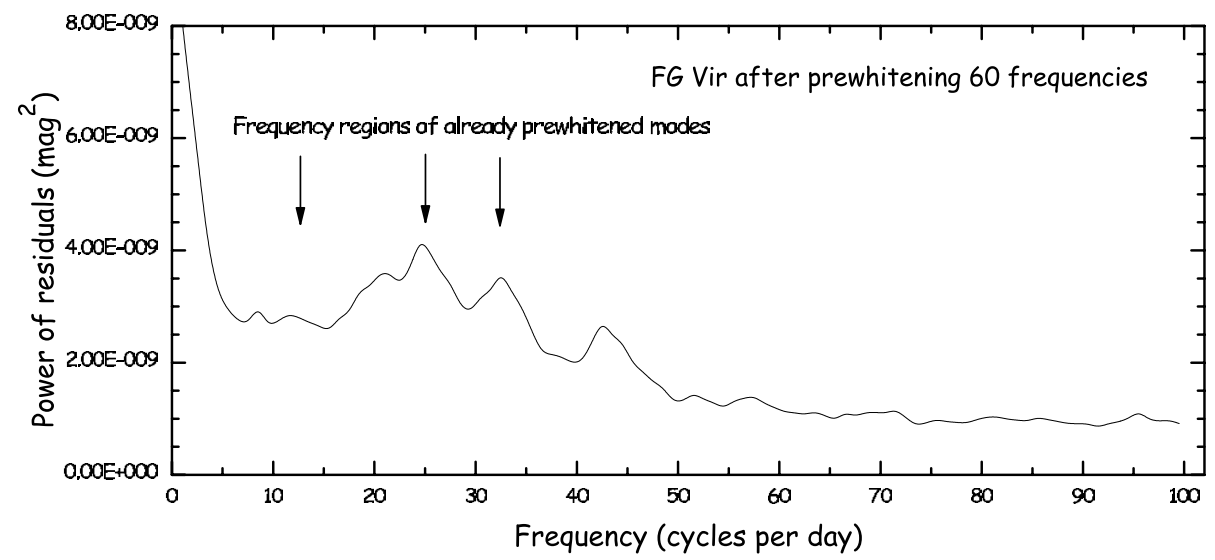

Figure 6. Power spectrum of the residuals of FG Vir after 60 frequencies were prewhitened. There exists excess power in two of the three main regions in which the prewhitened modes were found. This also suggests the presence of additional, so far undetected modes.

\section{Problems that need to be considered}

The recent developments in the study of these pulsating stars have shown a number of exciting discoveries as well as problems which need to be considered in future investigations from space or from the ground.

\subsection{Dense frequency spectrum requires exact model predictions}

An important tool to understand stellar structure is to compare the observed pulsation frequencies with those given by model calculations. As more frequencies as well as 


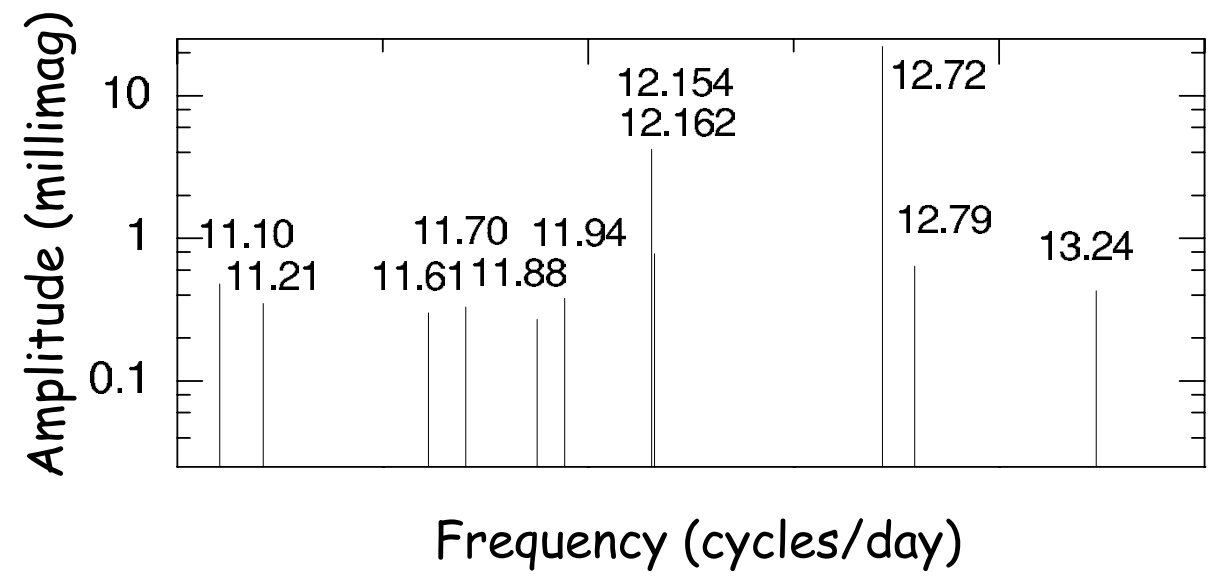

Figure 7. A very small frequency region for FG Vir, showing the dense observed frequency spectrum. This diagram demonstrates that pulsation models need to predict the pulsation frequencies to very high precision to enable unique comparisons with observations.

pulsation modes are identified, additional parameters (such as convective overshooting) can be finetuned in the models. However, the dense frequency spectrum observed leads to a uniqueness problem in the comparison. This can only be solved if the models can predict frequencies to much better than $1 \%$.

Let us use FG Vir again as an example. Fig. 7 shows a small frequency region with the detected frequencies. Unpublished spectroscopic as well as photometric mode-identification techniques have identified three of these modes with different values of $\ell$. We can identify the modes at $12.15,12.72$ and $12.79 \mathrm{~cd}^{-1}$ with $\ell=0,1$, and 2 , respectively. The nature of the $12.16 \mathrm{c} \mathrm{d}^{-1}$ mode and the other frequencies is unknown.

The dense frequency spectrum indicates that the pulsation models need to make very accurate frequency predictions to avoid misidentifications!

\subsection{Extremely close frequency pairs}

The majority of well-studied $\delta$ Scuti stars show a number of very close frequencies with separations as small as $0.01 \mathrm{c} \mathrm{d}^{-1}$. For the star BI CMi at least four close frequency pairs were discovered. It could be shown (Breger \& Bischof 2002) from amplitude and phase arguments that these were independent modes, rather than artefacts of the amplitude variability of a single mode. This may be related to the Blazhko Effect observed in another group of pulsators, the RR Lyrae stars.

Another close frequency pair can also be seen in Fig. 7, where the $12.154 \mathrm{c} \mathrm{d}^{-1}$ and $12.162 \mathrm{c} \mathrm{d}^{-1}$ modes for FG Vir are shown.

The close frequencies still need to be explained theoretically. On the observational side, they demand very high frequency resolution in the measurements (from space or ground), requiring observing runs longer than $100 \mathrm{~d}$.

\subsection{Beware of pulsation from companions}

Successful asteroseismology requires the matching between observed and predicted frequencies. It would be fatal if some of the observed frequencies would not belong to the star studied, but to a companion. In some cases, both components of a binary system pulsate, sometimes with similar periods, if their masses are similar. An example is the star $\theta^{2}$ Tau shown below in Fig. 8 (Breger 2005). 


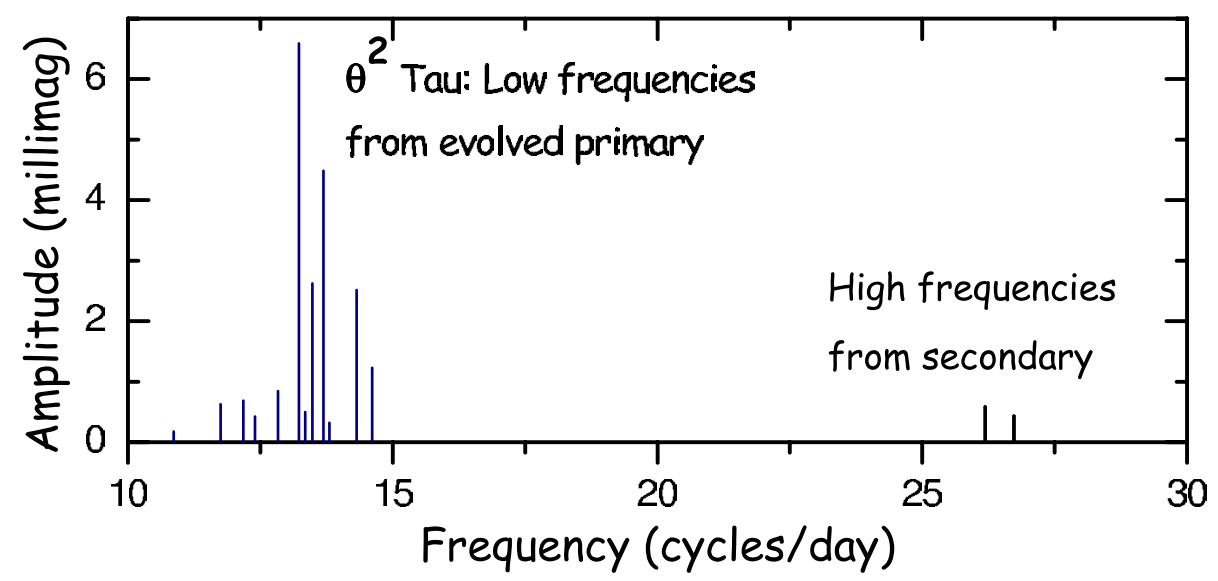

Figure 8. Example of a binary system, $\theta^{2}$ Tau, in which both components pulsate. The low and the high frequency groups show opposite light-time (O-C) shifts, which agree with those predicted from the known orbits. This diagram demonstrates that before modelling pulsations, one needs to ensure that all of the observed modes originate in the same star.

\subsection{Rotational splitting is not equidistant}

Even for moderate stellar rotation, rotational splitting is not equidistant (e.g., see a discussion by Goupil et al. 2000). This departure from equidistant splitting is very complicated to compute and provides a powerful tool when observations are compared with various stellar models. While rotational splitting has been easily observed in g-mode pulsators such as white dwarfs, for main-sequence A stars the theoretical complexity is matched by the difficulty of obtaining mode identifications for a complete $\ell=1$ triplet, or a $\ell=2$ quintuplet.

A triplet has now been detected. Fig. 9 shows an observed $\ell=1$ triplet for FG Vir. We confirm that rotational splitting is indeed nonequidistant.

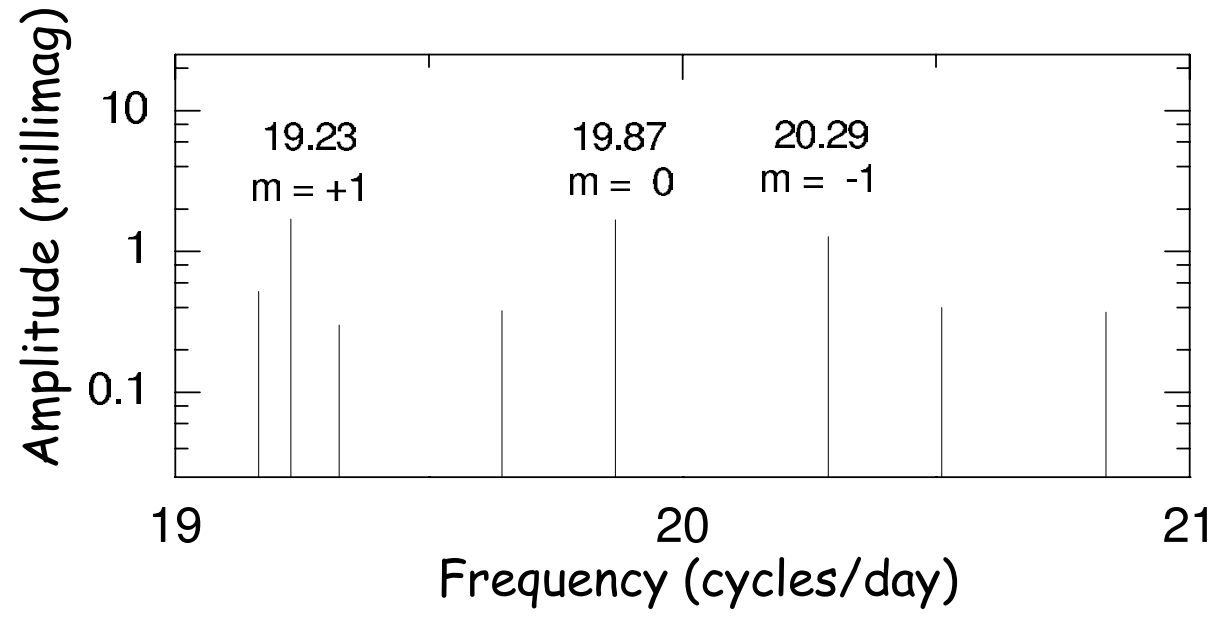

Figure 9. Example of observed non-equidistant rotational splitting of a $\ell=1$ triplet in FG Vir. 


\section{Acknowledgements}

This investigation has been supported by the Austrian Fonds zur Förderung der wissenschaftlichen Forschung, project number P17441-N02.

\section{References}

Aerts, C., Cuypers, J., De Cat, P., Dupret, M. A., De Ridder, J., Eyer, L., Scuflaire, R., Waelkens, C. 2004, AछึA, 415, 1079

Breger, M. 2005, ASP Conf. Ser., in press (Granada Workshop)

Breger, M. \& Bischof, K. M. 2002, AËA 385, 537

Breger, M. \& Pamyatnykh, A. A. 2002, ASP Conf. Ser. 259, 388

Breger, M., Pamyatnykh, A. A., Pikall, H. \& Garrido R. 1999, A\& A, 341, 151

Breger, M., Garrido, R., Handler, G., Wood, M. A., Shobbrook, R. R., Bischof, K. M., Rodler, F., Gray, R. O., Stankov, A., Martinez, P., O'Donoghue, D. O., Szabo, R., Zima, W., Kaye, A. B., Barban, C., Heiter, U. 2002, MNRAS, 329, 531

Buzasi, D. L., Bruntt, H., Bedding, T. R., Retter, A., Kjeldsen, H., Preston, H. L., Mandeville, W. J., Catanzarite, J., Conrow, T., Laher, R. 2004, ApJ, in press

Dupret, M. A., Grigahcéne, A., Garrido, R., Gabriel, M., Scuflaire, R. 2004, A\&A, 414,1081

Goupil, M.-J., Dziembowski, W. A., Pamyatnykh, A. A., Talon, S. 2000, ASP Conf. Ser., 210, 267

Guzik, J. A., Kaye, A. B., Bradley, P. A., Cox, A. N., Neuforge, C. 2000, ApJ, 542, L57

Poretti, E., Garrido, R., Amado, P. J., Uytterhoeven, K., Handler, G., Alonso, R., Martín, S., Aerts, C., Catala, C., Goupil, M. J. 2003, A\&\&A, 406, 203

Zima, W., Kolenberg, K., Briquet, M. \& Breger, M. 2003, CoAst, 144, 5

\section{Discussion}

MoEHler: You mentioned the detection of variability at the $1-2$ mmag level as an indication that indeed all stars in the variability strip vary. Are there any plans to study stars outside the instability strip at comparable precision to verify that they do not vary?

BREGER: Indeed, the space missions plan to investigate stars outside the classical instability strip. We already know that on both sides of the $\delta$ Scuti strip there are pulsators: the $\gamma$ Doradus gravity-mode pulsators to the cool side and isolated variables to the hot side.

WeIss: Within the COROT Additional Programme it is planned to test the hot and cool borders of the instability strip. This is also an on-going project for MOST.

BALOnA: The drop in the number of $\delta$ Scuti stars with rotation, as detected by photometry may be because the rapidly rotating stars pulsate with modes of high degree, which cannot be detected by photometry. The example of $\beta$ Pic comes to mind. 